\title{
A HANDHELD MICRODISCHARGE SPECTROSCOPY SYSTEM FOR HIGH-SPEED CHEMICAL ANALYSIS OF GASEOUS AND LIQUID SAMPLES
}

\author{
Bhaskar Mitra ${ }^{1}$, Brandon Levey, Tze-Ching Fung, Yogesh B. Gianchandani \\ Department of Electrical Engineering and Computer Science \\ University of Michigan, Ann Arbor, USA
}

\begin{abstract}
This paper presents a handheld microsystem that uses discharge spectroscopy to analyze chemicals both in vapor and liquid phases. The system employs a battery-operated circuit and interface for generating the microdischarge and performing the analysis. It uses swappable liquid and gas discharge microchips, which interface to the common platform. A pump and inert carrier gases are not utilized. The system can generate one or a series of single shot microdischarges per chemical analysis. Synchronized emission spectroscopy is used to optimize sampling. The liquid discharge microchip has an active area of $1 \mathrm{~mm} \times 1$ $\mathrm{mm}$ and a discharge gap of $50 \mu \mathrm{m}$. It employs a porous cathode which for introduction of the liquid into the microplasma. This results in a reliable system that can detect $2 \mathrm{ppm}$ of $\mathrm{Cr}$ sample without preconcentration. The gas discharge microchip uses the discharge between two metal electrodes. It has a discharge gap of $50 \mu \mathrm{m}$ and and an active area of $500 \mu \mathrm{m} \times 500 \mu \mathrm{m}$. The sensor detects 13 ppm of acetone vapor in air.
\end{abstract}

\section{INTRODUCTION}

In today's world of global terrorism and environmental pollution, there is increasing need for point-of-use chemical analysis. Conventional devices like chromatographs and mass-spectrometers must be complemented by fast, efficient, flexible, and portable devices. Miniaturized microsensors including SAW devices, chemi-resistors, chemi-capacitors, chemi-mechanical sensors, and metal oxide sensors which measure change in properties of thin films have been demonstrated for sensing of toxic vapors [1]. ISFETs, amperometric and potentiometric sensors, which measure changes in electrochemical properties of the solution have been developed for chemical sensing in the aqueous environments [2]. However, inspite of some excellent progress over the years, their use remains limited to specific chemicals in controlled environments. This is primarily due to the drifts in thin film properties and the difficulty in making stable miniature reference electrodes.

Microdischarge-based sensors are promising in this regard as they detect chemicals based on their atomic structure rather than their chemical properties. Although discharge spectroscopy in the form of ICPAES is one of the most sensitive techniques used in laboratories by chemists [3], discharge based sensors have not been used as microsensors. Many groups (including ours) have miniaturized microplasmas to chip scale for chemical sensing and other applications [4]. Many different kinds of powering schemes have been reported, including D.C. [5],
R.F. [6], inductively coupled plasmas [7], microwave discharges, and hollow cathode discharges [8]. Amongst these, D.C. or pulsed microdischarges are most suitable for use in handheld systems because they require simple circuitry and can be operated at atmospheric pressure in air ambient [9-12].

In previous work we have demonstrated low power microdischarge gas/vapor sensors [10],[12], and liquid electrode devices for the detection of trace elements in water samples [9],[11]. In this work, we present the first complete system that is handheld, and can also analyze both gaseous and liquid samples. The system employs disposable gas and liquid detection microchips (GDM and LDM) with a common interface. The GDM is based on a previous design [12], but has never been integrated into a system. The LDM is based on a new design and employs porous electrodes for sample delivery. This design permits controlled uptake of small volumes of the liquid sample and can be used to detect very low concentrations of metallic impurities in water samples. Both chips use atmospheric pressure pulsed microdischarges and avoid the use of a pre-concentrator to eliminate pumping demands.

Section II describes the design of the system and its components. Section III describes the fabrication process and Section IV describes the fabrication and experimental results.

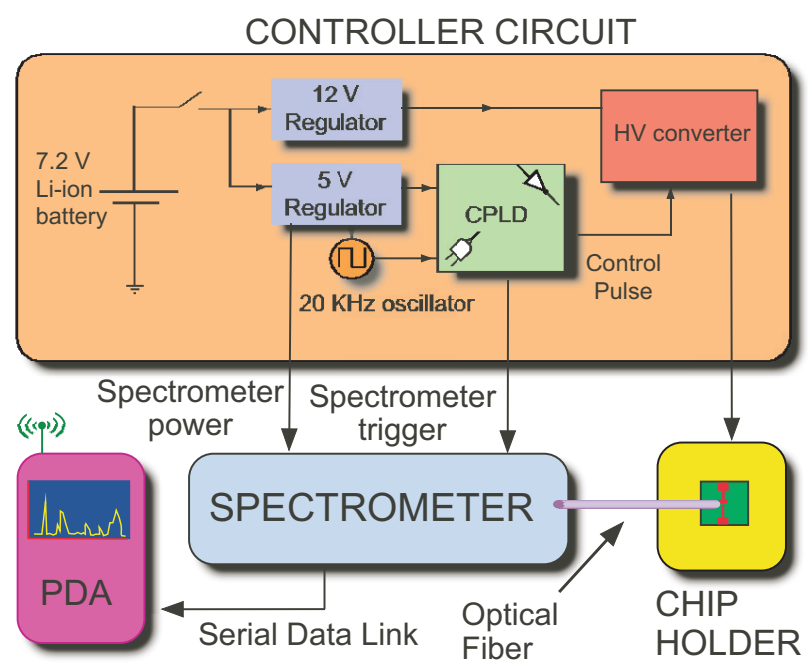

Fig.1: Schematic of the handheld system. The chip is placed in the chip holder and the discharge is struck using the control circuit. The spectrometer captures the optical signal and transfers the data to a wireless enabled PDA on a serial link. Inset: The controller circuit. 


\section{DESIGN}

The handheld system (Fig. 1) includes a sensor chipholder, a pager-sized spectrometer (USB 2000 from Ocean Optics), a PDA (HP IPAQ HX 2000 Pocket PC), and a battery operated control circuit and power management unit. The sensor chip-holder can accommodate both liquid and gas microdischarge chips, which can be easily swapped in and out. This is done by mounting the chips on a PCB and making contact to it by spring loaded contacts in the customized chip holder unit. The sensor chip holder also has a strategically placed collimating lens for coupling the microdischarge emission to an optical fiber (which connects to a spectrometer). This arrangement provides easy optical alignment and maximum light transfer to the optical fiber.

A custom circuit implemented as a programmable logic device provides up to two pulses of adjustable pulse duration (and delay between them) and two trigger signals with fixed duration, but variable delay. The system can accommodate more complex three electrode discharges and timing schemes also. In the current series of experiments, however, only two-electrode discharges are tested, which require one pulse and one trigger signal. The high voltage required for the microdischarge is provided by a programmable D.C.-D.C. converter (Pico HVP2P), which is powered by a $7.5 \mathrm{~V}$ battery and a $12 \mathrm{~V}$ regulator. The discharge pulse can be $1-400 \mathrm{~ms}$ long and $100-1500 \mathrm{~V}$ in amplitude. A control pulse from the CPLD (Atmel ATF1508AS) controls the timing and duration of the discharge pulse. The amplitude of the discharge pulse is controlled by varying the amplitude of the control pulse by using a potentiometer connected as a resistor divider. Figure 2 shows a timing diagram of the important pulse parameters. Two modes of operation are available: a single shot mode in which one microdischarge pulse is generated whenever the user presses a button; and a continuous mode, in which the device operates at a programmed rate. In both cases, the data from each microdischarge is captured and transferred to wireless enabled PDA at each discharge event.
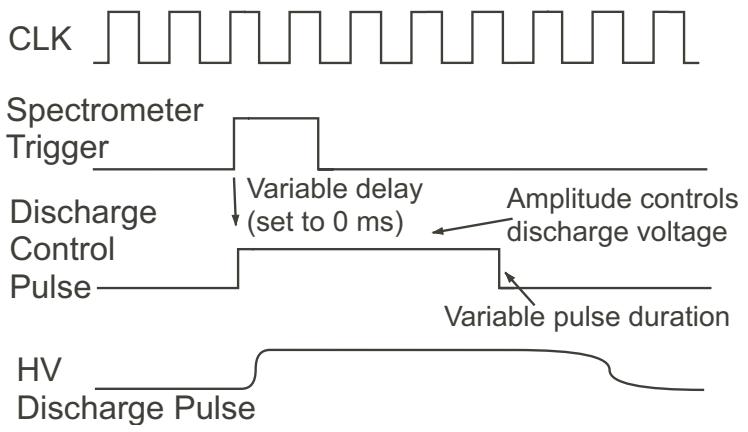

Fig. 2: Timing diagram for the single shot microdischarge. The optional second trigger and second control pulse is not shown.

The GDM employs a two electrode hybrid arc-plasma microdischarge [12] struck between two copper electrodes.
The highly energetic electrons in the plasma cause the organic vapors to disintegrate into smaller fragments, including many mono and diatomic fragments, which have emission in the UV-visible regions. At anode-cathode (A$\mathrm{K})$ gaps of $75 \mu \mathrm{m}$ or less, the discharge takes the form of a hybrid, consisting of a plasma glow over the cathode and an arc between the anode and the plasma. This discharge is used because it has a wide spectrum in energy for chemical analysis.

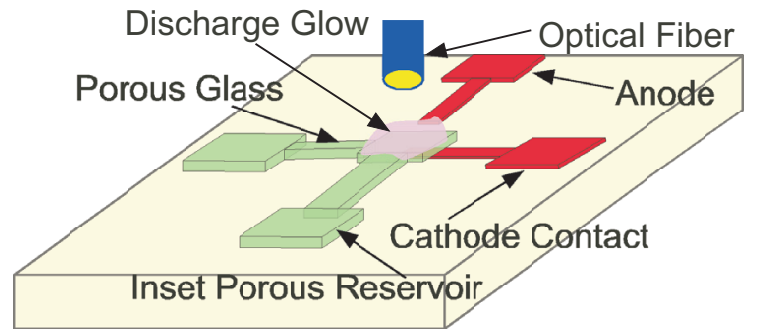

Fig. 3: Schematic of the liquid microdischarge chip.

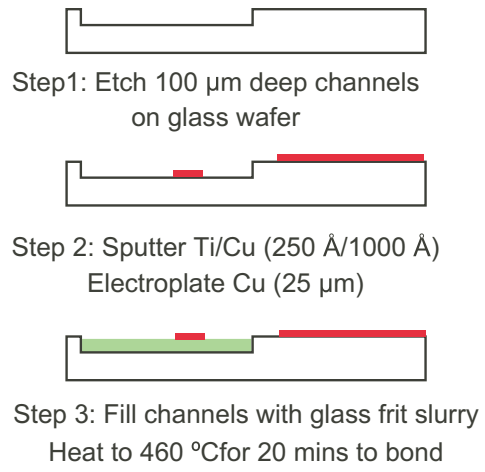

Fig. 4: Process for the fabrication of the liquid microdischarge chip. The fabrication for the gas discharge microchip involves only step 2 .

In the LDM (Fig. 3) a microdischarge is struck between a metal anode and a porous glass cathode. The porous cathode is wetted by the liquid sample, which is added through a side port in the chip. When plasma is struck between the metal impurities in the wet electrode are sputtered into the discharge and undergo atomic transitions in the plasma, which have characteristic line spectra in the plasma glow. The porous cathode allows for controlled uptake of the liquid sample, which eliminates splashing of the liquid, which increases device reliability at smaller A-K gaps. The smaller A-K gaps also enable the use of atmospheric pressure microplasmas rather than arcs. It is notable that microplasmas are driven, to a large extent, by secondary emission from the cathode so the use of the cathode as a means of sample delivery results in a very sensitive device.

\section{FABRICATION}

Both the devices use electroplated copper as the metal electrodes. For the GDM, a blanket Ti/Cu $(50 \mathrm{~nm} / 100 \mathrm{~nm})$ 
sputtered deposition acts as the base layer. A layer of photoresist (Clariant, AZ9260) is used to define a $50 \mu \mathrm{m}$ thick mold for the plating. A layer of copper, $25 \mu \mathrm{m}$ thick, is then electroplated through the mold to define the electrodes. The photoresist and the base layers are then etched away to leave the electrode structure intact.

The LDM fabrication (Fig. 4) begins with a glass-etch to define the porous cathode. A layer of $\mathrm{Cr} / \mathrm{Au}(50 \mathrm{~nm} / 500$ $\mathrm{nm}$ ) is used as the etch mask. Then the copper electrodes are electroplated, as in a GDM, to form the anode and cathode contact respectively. Finally the porous cathode is fabricated by filling channels with glass frit slurry in acetone $(0.2 \mathrm{~g} / \mathrm{ml})$, followed by sintering in a furnace at $460^{\circ} \mathrm{C}$ for $20 \mathrm{mins}$.

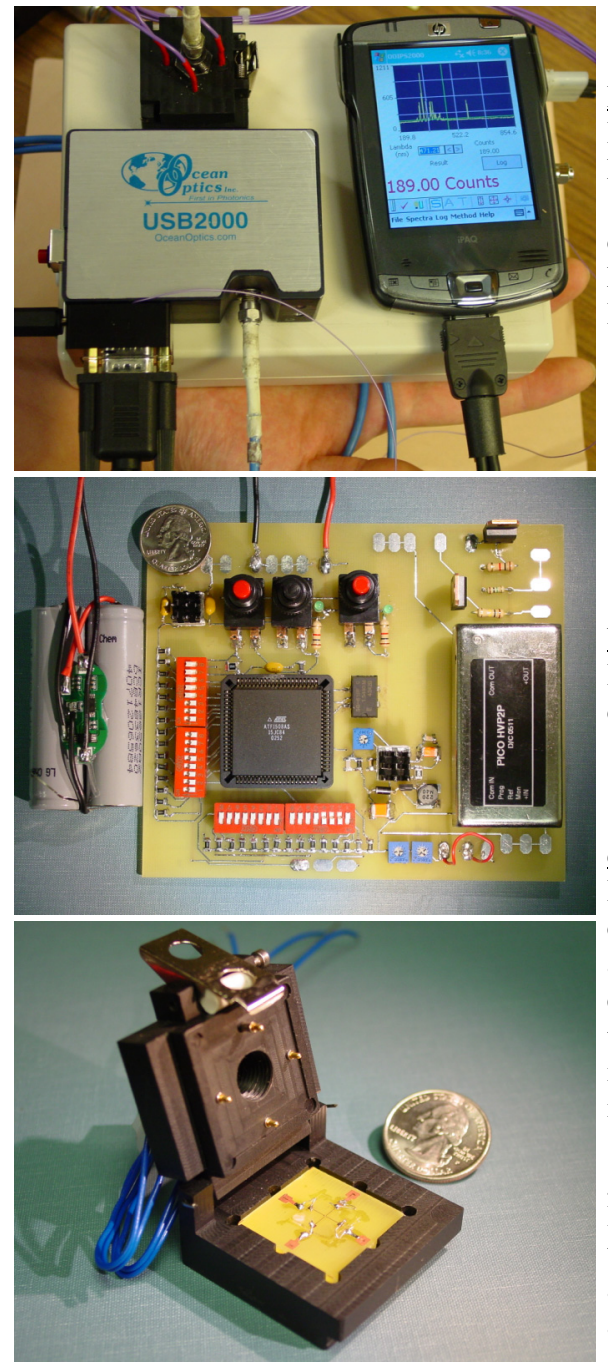

Fig. 5a (top): Photograph of the handheld system showing the controller, the chip holder and the PDA.

b (middle):

Picture of the controller circuit.

\section{c (bottom):}

Photograph of the chip holder shown against a US quarter. Contact to the terminals is made mechanically by a spring loaded, gold plated pin. A lens is placed in the middle, aligned with the active area.

\section{RESULTS}

The handheld system and its components are shown in Fig. 5. The overall dimensions of the system are $19.2 \mathrm{~cm} x$ $12.7 \mathrm{~cm} \times 4.3 \mathrm{~cm}$, and can be made smaller if desired. The handheld spectrometer, with a range of $200-850 \mathrm{~nm}$ is powered by the battery operated circuit.

Figure 6 shows a picture of the fabricated LDM and GDM chips against a US dime. The green color of the porous cathode is due the copper-oxide additives in the glass frit. The active area of the LDM is $1 \mathrm{~mm} \times 1 \mathrm{~mm}$ with an A-K gap of $50 \mu \mathrm{m}$. The GDM has an active area of 500 $\mu \mathrm{m} \times 500 \mu \mathrm{m}$ and an A-K gap of $50 \mu \mathrm{m}$.

A standard pulse of amplitude $850 \mathrm{~V}$ and duration $4 \mathrm{~ms}$ was used for all testing. A $10 \mathrm{k} \Omega$ resistor is ballast resistor was connected to ground to regulate the discharge.

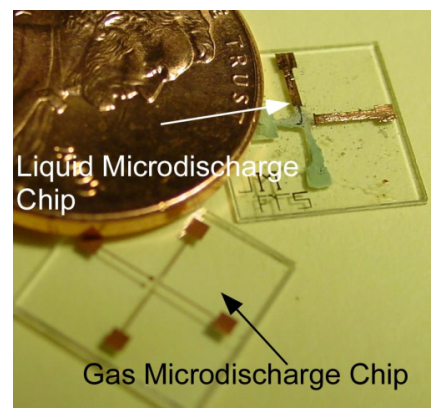

Fig. 6: Picture of the liquid and gas microdischarge chip shown against a US penny. The glass frit is green in color due to the presence of copper oxide.

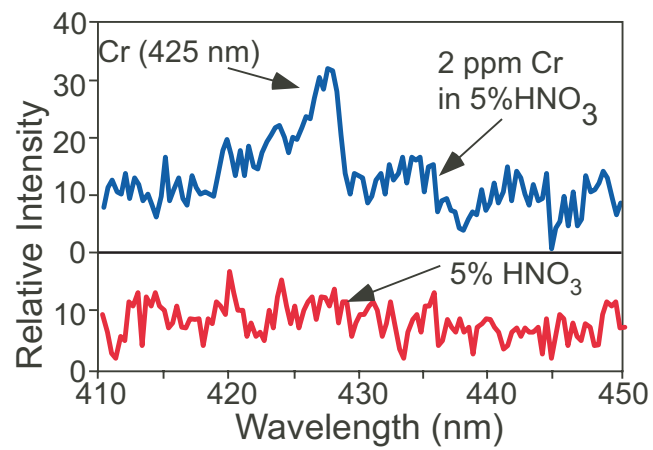

Fig. 7: Spectra from the liquid spectroscopy microchip (obtained without pre-concentration). A $425 \mathrm{~nm}$ line due to chromium (top) is clearly seen. The control sample (bottom) shows no emission at that wavelength.

For evaluating the LDM, a Cr plasma standard in 5\% $\mathrm{HNO}_{3}(2 \mathrm{ppm})$ was used as benchmark. A $1 \mu \mathrm{L}$ sample was loaded through the side port which was wicked into the porous electrode. The sample was loaded only once to avoid preconcentration effects. Figure 7 shows the captured spectrum, having emission from the $425 \mathrm{~nm}$ Cr line which is missing from the control sample of $5 \% \mathrm{HNO}_{3}$. The sensitivity of the chip can be further increased by using preconcentration. In such a mode the sample is loaded and let dry. If done repeatedly the metal salt accumulates in the cathode and hence gives a stronger signal. Another consequence of preconcentration is that the $\mathrm{pH}$ gradually increases due to acid accumulation. It is well known that the microdischarge device shows better performance at 
lower $\mathrm{pH}$ [9], so this helps getting better sensitivity without using a highly acidic sample.

For evaluation of the GDM, the chip holder was placed in a controlled vapor environment where the concentration of acetone was maintained at $13 \mathrm{ppm}$. Figure 8 shows the spectra in acetone and air ambient. The $385 \mathrm{~nm}$ line is present in acetone but absent in air.
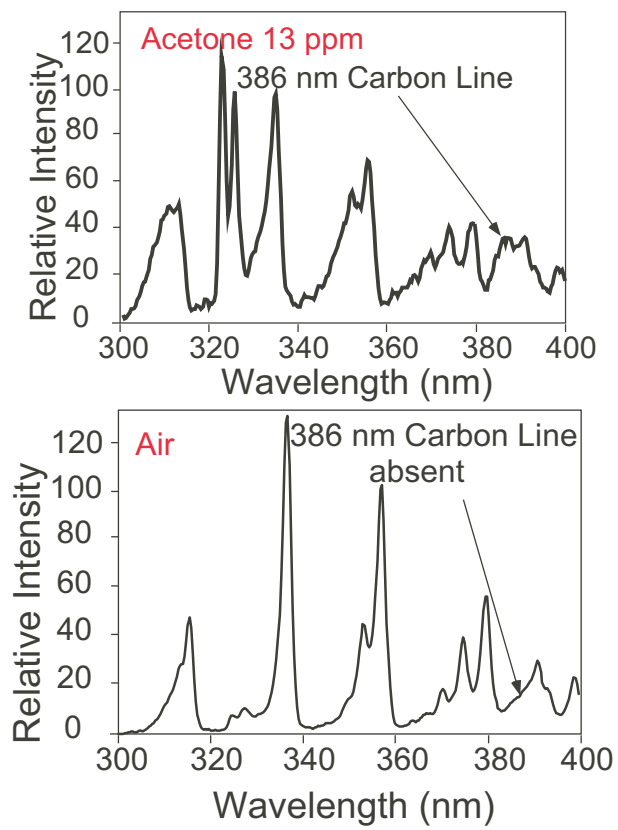

Fig. 8: Spectra from the gas discharge microchip (w/o preconcentration) (a-top) in $13 \mathrm{ppm}$ acetone showing the presence of $385 \mathrm{~nm}$ carbon line. (b-bottom) Control experiment in air. The $385 \mathrm{~nm}$ line is absent.

\section{CONCLUSION}

Sensors based on microdischarge spectroscopy are promising because they can detect a wide range of chemicals almost instantaneously. However, miniaturizing these devices has been a challenge due to power and reliability concerns. This work demonstrates the possibility of a handheld microdischarge based chemical analysis system for sensing in gaseous and liquid environments. The system employs swappable gas and liquid microdischarge chips and a chip holder which interfaces to both providing a low impedance contact and automatic optical alignment to the discharge. The microdischarges operate at atmosphere in air ambient, eliminating the need for a pump or special gases for the analysis. The gas discharge microchip can detect $13 \mathrm{ppm}$ of acetone vapor in air ambient. The liquid discharge microchip demonstrates the first use of a wet porous cathode. The porous cathode introduces controlled amount of sample by wicking in liquid through the pores, while still providing a low impedance to the cathode contact. This eliminates splashing of the liquid, and increases device reliability and sensitivity over discharges, which employ a liquid electrode. Using this chip and the handheld system the detection of $2 \mathrm{ppm}$ of $\mathrm{Cr}$ in $5 \% \mathrm{HNO}_{3}$ is demonstrated.

\section{ACKNOWLEDGEMENTS}

This work is funded in part by funding from the National Science Foundation. The authors would like to thank Mr. Weibin Zhu for help with the fabrication.

\section{REFERENCES}

[1] D.S. Wilson, S. Hoyt, J. Janata, K. Booksh and L. Obando "Chemical sensors for portable, handheld field instruments," IEEE Sensors Journal, 1(4) pp. 256-274, Dec 2001

[2] J. Janata, "Electrochemical microsensors," Proc. of IEEE, 91(6), pp. 864 - 869, June 2003

[3] E.H. Evans, J.A Day, W.J. Price, C.M.M. Smith, K. Sutton, "Atomic spectrometry update. Advances in atomic emission, absorption and fluorescence spectrometry and related techniques," Journal of Analytical Atomic Spectrometry, 18(7), pp. 672-711, 2003

[4] V. Karanassios, "Microplasmas for chemical analysis: analytical tools or research toys? ," Spectrochimica Acta Part B, 59(7), pp. 909-928, July 2004

[5] J.C.T. Eijkel, H. Stoeri, A. Manz “A dc Microplasma on a Chip Employed as an Optical Emission Detector for Gas Chromatography," Anal. Chem., 72, pp. 2547-2552, June 2000

[6] J.A.C Broekaert, "The development of microplasmas for spectrochemical analysis," Anal. Bioanal. Chem., 374, 2002, pp. 182-187

[7] J. Hopwood, "A Microfabricated Inductively Coupled Plasma Generator," Journal of Microelectromechanical Systems, 9(3), pp. 309-313, 2000

[8] S.J. Park, J. Chen, C. Liu, J.G. Eden, "Silicon microdischarge devices having inverted pyramidal cathodes: fabrication and performance of arrays," Appl. Phys. Lett., 78, pp. 419-21, 2001

[9] C.G. Wilson, Y.B. Gianchandani "LEd-SpEC: Spectroscopic detection of water contaminants using glow discharge from liquid electrodes," IEEE Transactions on Electron Devices, 49(12), Dec 2002, pp. 2317-22

[10] B. Mitra and Y.B. Gianchandani, "The Micromachined FlashFET: A Low-Power, Three-Terminal Device For High Speed Detection of Vapors at Atmospheric Pressure," IEEE MEMS Conference, Miami, 2005

[11] L. Que, C. Wilson, and Y.B. Gianchandani, "Microfluidic Electrodischarge devices with integrated dispersion optics for spectral analysis of water impurities," Jour. of Microelectromechanical Systems, 14 (2), pp. 185191, April 2005

[12] B. Mitra and Y.B. Gianchandani "Microdischargemicroplasma hybrids for detection of vapors at atmospheric pressure," IEEE Sensors Conference, Irvine CA, Nov. 2005 